Math. Model. Nat. Phenom.

Vol. 5, No. 2, 2010, pp. 185-207

DOI: $10.1051 / \mathrm{mmnp} / 20105207$

\title{
Past, Present and Future of Brain Stimulation
}

\author{
J. Modolo ${ }^{1,3}$, R. Edwards ${ }^{2}$, J. Campagnaud ${ }^{3}$, B. Bhattacharya ${ }^{3}$, A. Beuter ${ }^{3 *}$ \\ ${ }^{1}$ Lawson Health Research Institute, Department of Medical Biophysics \\ University of Western Ontario, N6A 4V2 London, ON Canada \\ ${ }^{2}$ Department of Mathematics and Statistics, University of Victoria \\ V8W 3R4 Victoria, B.C. Canada \\ ${ }^{3}$ Laboratoire IMS, Site ENSCPB, Institut Polytechnique de Bordeaux \\ 16 avenue Pey-Berland, 33607 Pessac, France
}

\begin{abstract}
Recent technological advances including brain imaging (higher resolution in space and time), miniaturization of integrated circuits (nanotechnologies), and acceleration of computation speed (Moore's Law), combined with interpenetration between neuroscience, mathematics, and physics have led to the development of more biologically plausible computational models and novel therapeutic strategies. Today, mathematical models of irreversible medical conditions such as Parkinson's disease (PD) are developed and parameterised based on clinical data. How do these evolutions have a bearing on deep brain stimulation (DBS) of patients with PD? We review how the idea of DBS, a standard therapeutic strategy used to attenuate neurological symptoms (motor, psychiatric), has emerged from past experimental and clinical observations, and present how, over the last decade, computational models based on different approaches (phase oscillator models, spiking neuron network models, population-based models) have started to shed light onto DBS mechanisms. Finally, we explore a new mathematical modelling approach based on neural field equations to optimize mechanisms of brain stimulation and achieve finer control of targeted neuronal populations. We conclude that neuroscience and mathematics are crucial partners in exploring brain stimulation and this partnership should also include other domains such as signal processing, control theory and ethics.
\end{abstract}

Key words: brain stimulation, spiking neurons, neural field models AMS subject classification: 92B20, 82C32

\footnotetext{
${ }^{*}$ Corresponding author. E-mail: beuter@idc.u-bordeaux2.fr
} 


\section{Brief history of deep brain stimulation and modeling ap- proaches}

\subsection{Brief historical development}

Brain stimulation is defined as

"Non-invasive and invasive techniques, and technologies that alter brain function through the use of electrical, magnetic, radiowave, or focally targeted pharmacological stimulation."

according to Brain Stimulation, a journal first published in 2008. However, work on brain neuromodulation started long before the publication of this journal.

The effects of electrical stimulation of the nervous system to treat human disease have long been recognized starting in Ancient Rome when Scriborius (15 AD) discovered that pain could be relieved by contact with a torpedo fish [16]. Centuries later, Franklin (1774) discovered, and then Bartolow [5] in 1874 showed, that stimulating the human motor cortex electrically induced muscle contraction. In 1886, intraoperative electrical stimulation was used by Horsley to identify the thumb cortical area for local resection in a patient with focal epilepsy [52]. However, the first electric stimulator battery operated and designed for human disease treatment appeared only at the beginning of the $20^{t h}$ century. This was followed by the introduction of stereotactic techniques by Spiegel [44], and intraoperative stimulation in humans became critical for electrode localization prior to lesion production in deep brain structures [16]. Mapping cortical and subcortical structures and producing atlases (which are still used today) was made possible thanks to electrical stimulation.

The use of electrical stimulation as a therapeutic intervention developed slowly at first because high-frequency stimulation was avoided for security reasons and long-term stimulation was not technically possible because of the unavailability of implantable stimulators. However, the first reported therapeutic use of electrical stimulation for motor disorders appears in Russia [6]. The first area to benefit widely from these discoveries was the area of pain, and the first stimulator became available commercially in the late nineteen sixties. In the early seventies, various pain conditions including denervation facial pain, spasticity, multiple sclerosis, anesthesia dolorosa, started to benefit from electrical stimulation of deep brain structures such as the thalamus or the periventricular area [16]. The field of deep brain stimulation (DBS) was born. In parallel, focal electrical stimulation for epilepsy developed [1,29]. With the discovery by Benabid in the late eighties that high frequency stimulation $(>100 \mathrm{~Hz}$ ) mimics the effect of lesioning, new applications of DBS became available for Parkinson's disease but also dystonia, obsessive compulsive disease, cluster headaches, and experimental approaches are currently being performed in the field of obesity and food intake control [8]. Obviously, this is a growing field and new applications are to be expected in the near future.

One observation about these electrical therapeutic applications is that many issues concerning the effects of electrical stimulation of biological tissue appear common to all of these areas and thus findings obtained in one application might carry over to other applications. An especially 
interesting example is a chapter that was written by Winfree [55] entitled "Are Cardiac Waves Relevant to Epileptic Wave Propagation?" [32]. It starts with the following paragraph:

"The mechanisms of cardiac fibrillation and of the epilepsies differ markedly. Also, there are many kinds of fibrillation and many epilepsies. Yet some collective parallels stand out. Their tissue substrates (heart muscle, brain neocortex and sub-cortical nuclei) both involve electrically active cells connected to neighbors, and both aberrations are electrical modes that seem alternative to the normal modes in the same tissue without change of parameters. The onset and offset of each is an abrupt switch from or back to the normal mode, both can be evoked by electrical stimulation at $10-100 \mathrm{~Hz}$ and current density around $20 \mathrm{~mA} / \mathrm{cm}^{2}$, and both can be terminated by a brief stimulus. In the case of fibrillation a single harsh direct-current shock of several milliseconds' duration is in fact the only known acute therapy. In the case of epilepsy, Gluckman et al. (1996) report prompt suppression of epileptiform activity in hippocampal slices using much smaller $60 \mathrm{~Hz}$ AC fields, and it is well known that a single brief sensory stimulus given close to seizure onset often appears to abort it [653]. Both fibrillation and epilepsy look quite irregular electrically, yet both have dominant periodicities in the 3 to $10 \mathrm{~Hz}$ range, and both processes seem to be determined at least in part by propagation geometry on the scale of centimeters." (p.165).

Today, a significant portion of the literature is devoted to neurostimulation [56] and "neurodevices for the management of nervous system disorders have been recognized as most promising through the coming decades" ([10], p. 247). Indeed, the introduction of new technologies combined with intelligent continuous feedback control of pathological oscillations should bring continued progress in the field of DBS.

In the case of Parkinson's disease, as early as 1966, Jasper used microelectrodes to record electrical activity from single nerve fibres in the thalamus (caudal portion of the Ventralis Lateralis nucleus-VL) of patients with Parkinson's disease (PD $)^{\dagger}$. In parallel he also recorded the electromyogram (EMG) from selected muscles, and tremor (i.e., estimated via mechanical muscle displacement). He made fascinating observations: First, cells firing in bursts were precisely timed at the frequency of tremor (EMG) and started bursting just before tremor onset; second, in some cases the microelectrode recorded from two thalamic locations: one firing in bursts before flexor muscle contraction and the other firing during extensor muscle contraction; third, the same thalamic cells that fire in bursts with tremor can also be activated by voluntary movements of the same limb and these movements also arrest tremor. Jasper concluded that there was a definite relationship between the part of the thalamus involved in tremor and voluntary movement. Furthermore, the optimal target found empirically to stop tremor by stereotactic ${ }^{\ddagger}$ neurosurgical lesion lies according to Jasper at the junction of the VL and the VP (Ventralis Posterior) in the Schaltenbrand and Bailey atlas. This target is relatively equivalent to the region denoted by other acronyms such

\footnotetext{
${ }^{\dagger} \mathrm{PD}$ is a neurodegenerative disorder characterized by tremor, rigidity, bradykinesia and postural instability.

‡The stereotactic method was first developed for animal experimentation and later adapted for human brain surgeries for locating points within the brain using an external 3-dimensional frame of reference and a Cartesian (threeorthogonal axis) system.
} 
as the VPLo proposed by Olszewski [37] or the Vim proposed by Hassler et al. [20]. Jasper's work was the first to our knowledge to demonstrate a link between activity of single neural cells and a parkinsonian symptom (i.e., tremor).

When Jasper published his work more than 40 years ago, functional neurosurgery for PD was already performed. Initially, it was done on open head and later on closed head using a stereotactic frame. Functional neurosurgery is defined by Speelman and Bosch as a neurosurgical procedure used to improve symptoms by making small lesions or by applying neuromodulation of the brain, spinal cord or nerve [43]. Early surgical operations for PD were attempted in multiple locations including extirpation of premotor and motor zones of the cortex, section of the pyramidal tract at various levels, lesion of GPi (internal part of the globus pallidus), lesion of VL, clipping of the anterior choroidal artery, extirpation of the head of the caudate nucleus, lesion of the ansa lenticularis, etc. (See [27], chapter 6 for details). During early surgeries no human brain atlas was available, as the first one was introduced by Spiegel and Wycis in 1952 [45]. More recently, new imaging techniques have become available and complement information provided by brain atlases.

Many of these surgeries decreased at the end of the 60's after L-Dopa ${ }^{\S}$ was discovered. About 20 years ago, a resurgence of these surgeries occurred after the discovery of severe side-effects in patients taking L-Dopa for several years. However, instead of lesioning a target, neurosurgeons now usually perform a new neurosurgical procedure called Deep Brain Stimulation (DBS). In this treatment, permanent macroelectrodes, usually one on each side of the brain, are connected to a stimulator implanted in the chest wall of the patient. Chronic electrical stimulation produces an alleviation of symptoms similar to that obtained with a lesion of the same target. Usually, three parameters define the stimulation pattern: amplitude, pulse width and frequency; with the pulses being biphasic and charge-balanced to avoid tissue damage. These stimulation pulses may be applied with monopolar or bipolar stimulation. One advantage of such a procedure is its reversibility. Chronic electrical stimulation of the thalamus (Vim) for tremor reduction in human subjects was first reported in 1987 by Benabid et al. [7]. In this study, continuous Vim stimulation using stereotactically implanted electrodes connected to subcutaneous stimulators strongly decreased tremor. Today, functional neurosurgery has become a standard procedure used to alleviate both movement disorders and psychiatric disorders, including PD, dystonia, epilepsy, depression, obsessive compulsive disorders, etc. [54]. Today, the number of patients with PD receiving DBS is estimated between forty and fifty thousand in the world.

While scientific studies and clinical applications of DBS have progressed regularly over the last decades, its underlying physiological mechanisms still remain unclear today. It has been suggested that neurological disorders such as pain, tinnitus $₫$, Parkinson's disease, or depression arise from cortical dysrhythmia [15] and more recently from thalamocortical dysrythmia [28]. Magnetoencephalographic recordings in human patients show that specific brain rhythms augment or decrease when symptoms are present and it has been hypothesized that, in some way, brain stimulation prevents resonant interactions (i.e., cooperation of neuronal assemblies around favoured frequencies) between brain structures. Thus, DBS may be seen as a way to correct abnormal cortical and sub-

\footnotetext{
$\S$ This medication, a precursor of the neurotransmitter dopamine, is able to cross the blood-brain barrier and to increase dopamine levels in the central nervous system for the treatment of PD.

"Hearing a sound in the absence of any external auditory stimulus.
} 
cortical rhythms occurring in PD (see [48] for an experimental study on abnormal cortical rhythms in PD).

One difficulty in understanding DBS mechanisms is to relate the rapid development of molecular and cellular neurobiology at one extreme, with human imaging data at the other [19]. Another limitation is that intuitive interpretation of dynamic brain processes is rarely sufficient [19]. To decipher the complex brain mechanisms underlying behavior in its normal and pathological manifestations, it is important to explore not only all relevant levels (molecules, cells, networks and behaviors) but also to integrate or link the processes occurring at these multiple scales. Specifically, in order to improve our understanding of brain mechanisms involved in DBS, it is necessary to bridge gaps across spatial (and also temporal) scales. This may be achieved by developing a close interaction between neuroscience and mathematical modelling [19]. While this must be done very carefully (see [51]), mathematical formalism offers a powerful and rigorous way to integrate levels of organization and make predictions. As illustrated in Fig. 1, the initial mathematical model of DBS appeared only 10 years ago [46] and involved phase oscillators. Over the last decade, other models have been published including among others an oscillator network model [50], a spiking neuron network model [41], and a population based model [33].

\begin{tabular}{|cccc|}
$\begin{array}{c}\text { phase oscillator } \\
\text { model }\end{array}$ & $\begin{array}{c}\text { oscillator network } \\
\text { model }\end{array}$ & $\begin{array}{c}\text { spiking neuron } \\
\text { network model }\end{array}$ & $\begin{array}{c}\text { population-based } \\
\text { model }\end{array}$ \\
\hline 1999 & 2001 & 2004 & 2008 \\
\hline Tass & Titcombe et al. & $\begin{array}{c}\text { Mclntyre et al. } \\
\text { Rubin and Terman }\end{array}$ & Modolo et al. \\
\hline
\end{tabular}

Figure 1: Evolution of a decade of modeling approaches.

In the present paper, we illustrate the close interaction existing between mathematics and neuroscience through a model based on neural field equations. This model is developped to control targeted cortical populations of neurons with the goal of attenuating symptoms more efficiently in PD.

\subsection{Modeling approaches over the last decade}

Approaching the problem of DBS physiological mechanisms from a mathematical perspective is challenging in several respects. The most obvious among these is that the brain consists of a huge number of inter-connected neural networks with complex cellular and wiring arrangements. Another problem is that neural elements (cellular bodies, axons) activated by DBS may differ depending on stimulation parameters. Nevertheless, several mathematical models have been developed since 1999 to provide further insight on how DBS achieves its therapeutic effects. These models are based on different mathematical approaches. Below, we review some of these approaches in the context of tremor treatment. 


\subsubsection{Phase oscillator model}

One of the pioneers in the domain of modelling the effects of DBS is Peter Tass, who has extensively studied phase oscillators and their potential medical application [46]. Briefly, a phase oscillator is defined only by its eigen frequency $\omega$ and its phase $\varphi(t)$. During an oscillation cycle, the phase varies from 0 to $2 \pi$, according to the following equation:

$$
\frac{\partial}{\partial t} \varphi(t)=-\omega
$$

Since the phase varies between 0 and $2 \pi$, the system state is often represented as a position on a circle. In the context of a network of $N$ phase oscillators coupled using a constant "synaptic strength" coefficient $K$, the equation for each phase oscillator $i$ interacting with other oscillators $j$ reads:

$$
\frac{\partial}{\partial t} \varphi_{i}(t)=-\omega_{i}+\frac{K}{N} \sum_{i=j}^{N} \sin \left[\varphi_{i}(t)-\varphi_{j}(t)\right]
$$

This is known as the Kuramoto model. By plotting the phase of each oscillator on a circle, one may observe whether oscillators are in phase synchrony (units tend to have the same position around the circle) or desynchronized (units are distributed in a uniform manner around the circle). The motivation for using this approach is that, in PD, the firing of neurons in several basal ganglia (BG) structures tend to become more synchronized. In this situation, the synchronization of neuronal firing appears to be deleterious for motor function (i.e., with the presence of rest tremor at the periphery). Consequently, modeling networks of coupled oscillators that may synchronize above a certain coupling threshold is appropriate for investigating how DBS modulates the synchronization of neural networks.

In his book, Tass presented new concepts related to the synchronization and desynchronization of phase oscillators [46]. More specifically, he studied the synchronization of phase oscillator networks subject to noise and external stimulation (response of a cluster of neurons to single stimulus, and periodic train of stimuli). Depending on the properties of the stimulus, the activity of a cluster of neurons could be dramatically affected, for instance by a phenomenon known as oscillator death (the oscillation amplitude vanishes). Also, he was the first to propose a feedback control of neuronal networks, i.e., to use the activity of the network to determine the stimulation signal. At the time, this concept was really novel, since until then (and even until now), therapeutic brain stimulation was performed only in open loop, where the state of the system is not taken into account to adapt the stimulation signal.

\subsubsection{Oscillator model}

One intriguing observation in studying the effects of DBS on rest tremor in PD is that, when DBS is turned "on", a delay occurs before tremor decays (typically, several seconds). However, using phase oscillator models, the desynchronization of the network, associated with the suppression of 
tremor, is immediate. To address this issue, a toy network of three oscillators with excitatory and inhibitory connections receiving high-frequency input was proposed [50]:

$$
\frac{d}{d t} y_{1}(t)=f_{I}\left(y_{3}\right)-y_{1}, \quad \frac{d}{d t} y_{i}(t)=f_{E}\left(y_{i-1}\right)-y_{i}, \quad i=2,3
$$

where $f_{E}$ and $f_{I}$ indicate excitatory or inhibitory coupling functions respectively. These coupling functions were assumed to be sigmoidal in form, and include a gain $g$ :

$$
f_{E}(y)=\frac{y^{g}}{y^{g}+\theta^{g}}, \quad f_{I}(y)=\frac{\theta^{g}}{y^{g}+\theta^{g}}
$$

To model the effect of DBS, Titcombe et al. assume that each pulse of DBS releases a small quantity $\delta$ of a substance (neurotransmitter for instance), the total quantity at time $t$ being $z(t)$. The hypothesis was that the quantity of this substance modified the gain parameter such that $g(t)=$ $g_{0}-z(t)$, with a decay $t_{c}$ leading to $\frac{d}{d t} z(t)=-\frac{1}{t_{c}} z(t)$. In this model, the gradual buildup and decay of $g$ leads to delays in onset and termination of tremor as observed clinically when DBS is turned off and on (see [50], Figs. 2 and 4). Despite this successful description of delayed therapeutic effect on tremor, one remaining issue was that the bifurcation parameter was not able to link physiological phenomena occurring at different scales of the system, which is necessary to complete the picture on how DBS works.

\subsubsection{Spiking neuron network model}

Early mathematical models of DBS effects were rather abstract (see above), even if some modeling work was based on a physiologically realistic description of how DBS affects the surrounding brain tissue [30]. However, in 2004 Rubin and Terman proposed a mathematical model [41] aiming at understanding the effects of DBS by modeling individual neurons located in several structures of the loop formed by the cortex, the basal ganglia and the thalamus (also called the "motor loop" because of its critical involvment in motor function). In an effort to accurately capture the specificity of neuronal activity in each structure, the authors based their model on variations of the HodgkinHuxley model [21], which is a comprehensive model of membrane potential variations with respects to external inputs and opening/closing of voltage-dependent ionic channels. By including in the model specific ionic channels found in the neurons, it is possible to realistically simulate their activity pattern. For instance, the set of modified Hodgkin-Huxley equations describing the activity of a subthalamic neuron is [47, 41]:

$$
C_{m} \frac{d}{d t} v(t)=-I_{L}-I_{K}-I_{N a}-I_{T}-I_{C a}-I_{G e \rightarrow S T N}+I_{D B S}
$$

where $C_{m}$ is the membrane capacitance, $I_{L}$ the leakage current, $I_{K}$ the potassium current, $I_{N a}$ the sodium current, $I_{T}$ the low-threshold calcium current, $I_{C a}$ the calcium current, $I_{G e \rightarrow S T N}$ the inhibitory synaptic input from the GPe-the external part of the globus pallidus, and finally $I_{D B S}$ accounts for the DBS current. It should be noted that every ionic current is voltage-, and thus time-dependent. In this model, as in a large majority of modeling studies, $I_{D B S}$ is supposed to 
have the same pulse profile as the current delivered by the electrode in the brain, even if diffusion of current through cerebral tissue and orientation of neurons are likely to complicate this picture.

Neurons from other structures (GPi, GPe, and thalamus) are modeled in a similar fashion, and these structures are then synaptically connected following a simplified scheme inspired from known neuroanatomy as shown in Fig. 2.

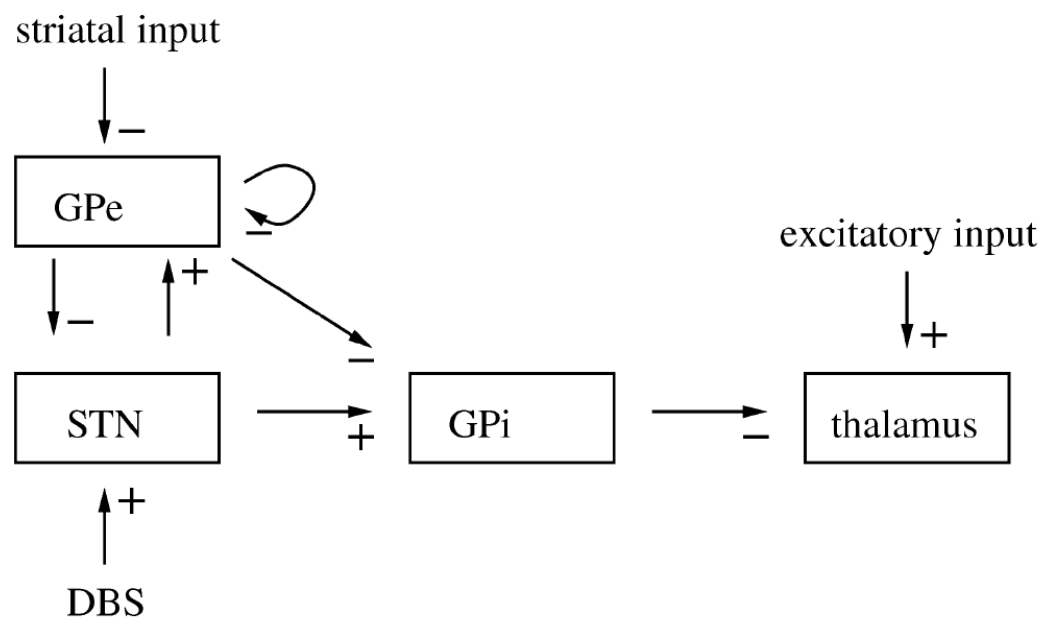

Figure 2: Architecture of the network of neuronal structures considered in [41] (with permission). GPe: external part of globus pallidus, STN: subthalamic nucleus, GPi: internal part of the globus pallidus, '+' denotes excitatory, '-' denotes inhibitory projections.

In this model, the switching from "normal" to "parkinsonian" states is simulated by increased striatal input to the GPe and decreased synaptic weights of intra-GPe inhibitory projections. In the normal state, both STN (subthalamic nucleus) and GPe have a weak, irregular and asynchronous activity, which enables thalamic cells to relay faithfully incoming sensorimotor signals to the cortex. Conversely, in the parkinsonian state, both STN and GPe have a strong, regular and synchronous activity at the tremor frequency (around $5 \mathrm{~Hz}$ ) which forces GPi cells to trigger $5 \mathrm{~Hz}$ bursts of spikes. This has a dramatic effect on thalamic cells: indeed, in this situation, thalamic cells are no longer able to transmit correctly incoming sensorimotor signals to the cortex. When DBS is applied to the STN model cells in the form of a monophasic square pulse at a certain frequency, amplitude and pulse width, it overrides pathological activity of STN and GPe cells. This means that low-frequency bursting is replaced by tonic firing with a $1: 1$ phase synchrony between STN activity and DBS pulses. This new pattern enables thalamic cells to resume relaying of sensorimotor signals to the cortex, thus the authors concluded that DBS achieves its therapeutic effects by increasing the firing rate of STN cells, rather than by decreasing it, which is not intuitive, because of the similarity in the clinical effects of STN stimulation and lesion.

This model represented a major advance in the modeling of DBS effects, since efforts were made to include realistic activity of neurons in several structures involved in motor control, but also to link the modulation of neuronal activity induced by DBS to functional consequences (performance of thalamic relay induced by DBS). 


\subsubsection{Population-based model}

Altough the model by Rubin and Terman [41] provided new insights into possible physiological mechanisms underlying DBS, it left several issues unclear, as highlighted in an extensive study of this model [39], such as: the issue of robustness with respect to parameter variation, complexity even in its simplified form, frequency-dependence of DBS therapeutic effects, decrease of STN neuron firing rate observed experimentally during DBS (see [31]) and effect of charge-balanced, biphasic stimulation pulses used in clinical practice.

Taking a different approach, a population based model of the complex formed by the STN and the GPe was developed [33, 35], which overcomes the above issues. Population based models have been extensively developed along the lines of statistical mechanics [36, 38], when the number of neurons in the population is very large (i.e., $N \rightarrow+\infty$ ). In this modeling approach, the focus is not on the individual state of every neuron or every synapse, but rather on the distribution of states $\rho(v, t)$ such that, in the population, the number of neurons with a potential in $[v, v+d v]$ is $\rho(v, t) d v$. This distribution obeys, in the most general form:

$$
\frac{\partial}{\partial t} \rho(v, t)=-\vec{\nabla} \cdot\left[F(v)+\mathbf{e}_{v} \sigma(t) \int_{v-\epsilon}^{v} \rho(\tilde{v}, t) d \tilde{v}\right]
$$

where $F(v)$ represents individual dynamics of a single cell (e.g., for a population of integrate-andfire neurons, $F(v)=-g\left(v-v_{L}\right)$ and $\vec{F}(\vec{w})$ for a model with several state variables where $\vec{w}$ is the state vector), $\sigma(t)$ is the mean field variable (i.e., the mean number of incoming spikes per cell per unit of time, assumed to be equal for all neurons of the population), $\epsilon$ is the amplitude of the excitatory post-synaptic potential (EPSP) caused by the activation of a single synapse (assumed to occur instantaneously), and $\mathbf{e}_{v}$ is a unit vector in the $v$ direction of the state space.

An application in a new context of population based models used the Izhikevich model [24] to take into account specific activity patterns exhibited (by STN or GPe neurons, for example). Using appropriate parameter values for the Izhikevich model, it is possible to use suitable expressions of $\vec{F}(\vec{w})$ that reproduce the main properties of STN and GPe neurons [33]. Then, it is possible to write the set of equations describing the network formed by the STN and GPe [33] thus:

$$
\begin{aligned}
\frac{\partial}{\partial t} \rho_{S T N}(\vec{w}, t) & =-\vec{\nabla} \cdot\left[\vec{F}_{S T N}(\vec{w})+\vec{e}_{V} \sigma_{S T N}(t) \int_{v-\epsilon}^{v} d \tilde{v} \rho_{S T N}(\vec{w}, t)-\vec{e}_{V} \sigma_{G P e}(t) \int_{v}^{v+\epsilon} d \tilde{v} \rho_{S T N}(\vec{w}, t)\right] \\
\frac{\partial}{\partial t} \rho_{G P e}(\vec{w}, t) & =-\vec{\nabla} \cdot\left[\vec{F}_{G P e}(\vec{w})+\vec{e}_{V} \sigma_{S T N}(t) \int_{v-\epsilon}^{v} d \tilde{v} \rho_{G P e}(\vec{w}, t)-\vec{e}_{V} \sigma_{G P e}(t) \int_{v}^{v+\epsilon} d \tilde{v} \rho_{G P e}(\vec{w}, t)\right]
\end{aligned}
$$

DBS was modeled as an input applied directly to the membrane of STN cells, appearing as an additive term in $\vec{F}(\vec{w})$. By varying the frequency of DBS, the response of STN cells showed a resonance phenomenon [33], explaining why DBS at low frequencies (around $20 \mathrm{~Hz}$ ) may worsen tremor. Furthermore, it was shown that the effect of charge-balanced, biphasic pulses of stimulation drastically reduced the response of STN cells $[33,35]$, highlighting the importance of a 
realistic stimulation pulse shape. Indeed, monophasic stimulation is purely excitatory, and thus increases the response of STN cells. This model was also used to propose a mechanism by which DBS reduces tremor, namely stimulation-induced functional decoupling (SIFD) [35], i.e., synaptic current influence becomes negligible during DBS, drastically reducing the neuronal coupling that gives rise to the undesirable low-frequency rhythm in the STN. Finally, another study in which the model was used to investigate the impact of cortical inputs on STN activity during DBS [34] concluded that the role of dopamine was to reduce neuronal coupling between the cortex and the STN, which was proposed to be a resonant pathway. This proposition based on model results was successfully verified in a study in which the EEG of PD patients receiving DBS in the presence or absence of dopamine was recorded [13]. Indeed, this paper showed that damping of oscillations in the STN induced by cortical inputs was stronger in the presence of dopamine.

While the models presented above were designed with the aim of improving our understanding of the physiological mechanisms underlying the clinical effects of DBS, the focus today is shifting to the conception of new stimulation patterns and targets based on mechanisms uncovered by previous studies. Today, although DBS has become a standard and efficient procedure, the limitations and side effects associated with this procedure are regularly pointed out by the scientific community. New stimulation patterns can be derived and tested from biologically realistic models or approaches, such as neural field models, presented in the following section.

\section{Neural field model}

\subsection{Background}

Neural field models provide a quantitative description of cortical activity in space and time. Historically, this modeling approach was developed in several famous papers $[53,2]$. They assume that, in the cortex for example, at a mesoscopic scale, neural masses (i.e., assemblies of about 1000 excitatory and inhibitory neurons, often referred to as the "cortical macrocolumn") are considered, and form a continuous network since somas and axons are densely packed. Such a continuous neural network, comprised of numerous neural masses, is called a neural field.

The variables used in neural field models are thus means of microscopic measures (e.g., of the membrane potential of individual neurons). Let $\psi(x, t)$ be the value of the neural field at position $x$ at time $t$, i.e., the mean potential of neurons forming the neural mass at position $x$. A general neural field equation reads $[2,3]$ :

$$
\hat{L}[\psi(x, t)]=\gamma \int_{\Omega} d y W(x-y) S\left[\psi\left(y, t-\frac{|x-y|}{c}\right)\right]+I(x, t)
$$

where $\hat{L}$ is a temporal operator (generally of first-order, $\hat{L}=\beta \frac{\partial}{\partial t}+1$, see [3]), $\gamma$ is the synaptic coupling strength, $\Omega$ is the spatial domain of the neural field, $W(x-y)$ is the connectivity function, $c$ is the spike transmission speed along axons, and $I(x, t)$ is an external stimulus. The connectivity function is assumed usually to be translationally invariant and gives the probability that neurons at a distance $|x-y|$ are connected. Depending on the connectivity kernel and on the presence of 


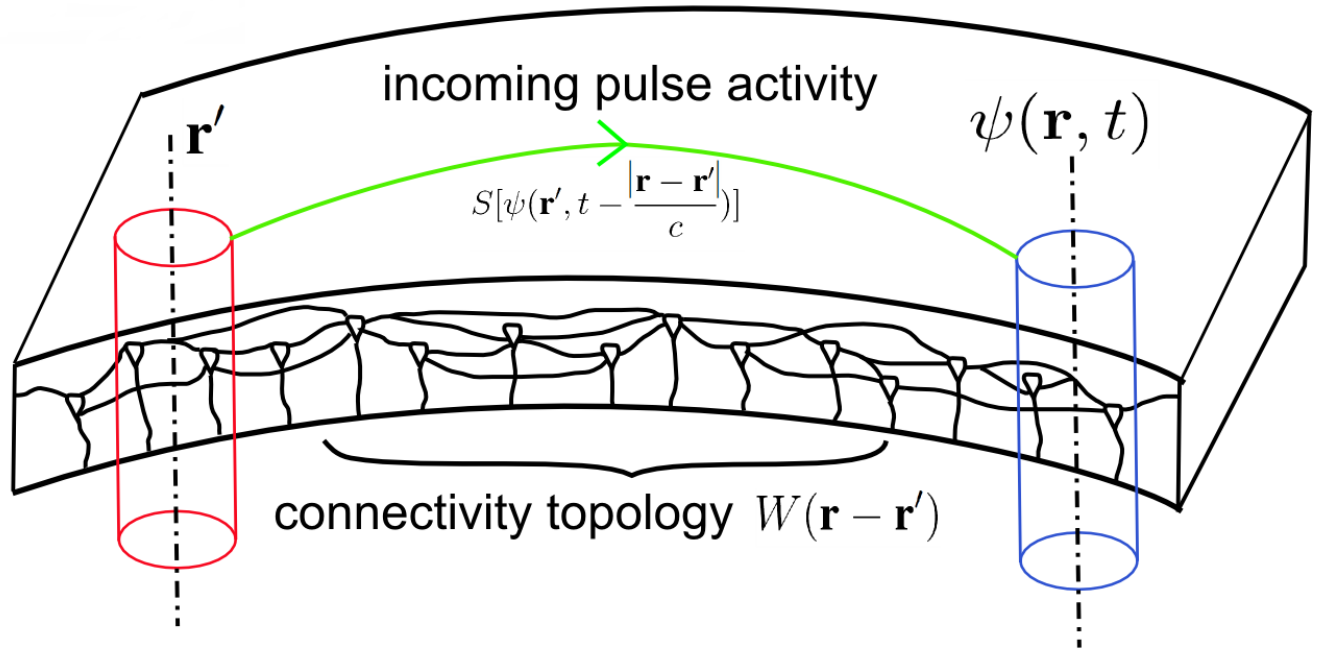

Figure 3: Schematic representation of cortical tissue with cylinders representing a local group of neurons (neural mass) used to derive the neural field equation. The neural field $\psi(\mathbf{r}, t)$ at each position $\mathbf{r}$ at time $t$ is expressed as a function of neuronal activity $S\left[V\left(\mathbf{r}^{\prime}\right), t-\frac{\left|\mathbf{r}-\mathbf{r}^{\prime}\right|}{c}\right]$ at other positions $\mathbf{r}^{\prime}$, where the conduction delay is $\frac{\left|\mathbf{r}-\mathbf{r}^{\prime}\right|}{c}$; and of connectivity topology $W\left(\mathbf{r}-\mathbf{r}^{\prime}\right)$.

time delays, different activity patterns may occur, such as traveling waves [3]. The neural field equation is derived using the fact that the synaptic response function $h$ describing the effect of a single synaptic event on the membrane potential, is a Greens function for the temporal operator $\hat{L}$,

$$
\hat{L} h\left(t-t^{\prime}\right)=\delta(t)
$$

where $t^{\prime}$ is the time at which the incoming spike caused synaptic activation, and $\delta(t)$ is the Dirac function. The synaptic response function is usually expressed as [3]:

$$
h\left(t-t^{\prime}\right)=\frac{\alpha \beta}{\alpha-\beta}\left[e^{-\alpha\left(t-t^{\prime}\right)}-e^{-\beta\left(t-t^{\prime}\right)}\right]
$$

where the parameters $\alpha, \beta$ characterize the shape of the synaptic response.

Neural field models can explain and predict dynamical phenomena of cortical activity, such as visual patterns during hallucinations [12], spiral waves [23] or traveling waves [42]. This class of models describes neuronal activity at a mesoscopic spatial scale, since the basic unit is the neural mass. Indeed, the cortical macrocolumn is a proposed basic pattern of cortical architecture, repeated a large number of times throughout the cortex. However, this notion is currently under criticism and it has been proposed that, even if it may represent a basic pattern of neuronal connectivity, it may not have a function by itself [22]. Instead, the interaction between neural masses would give rise to complex dynamical patterns of activity. Furthermore, since somas and axons are densely packed, it is assumed that the discrete network may be effectively approximated by a spatially continuous one. Finally, several temporal scales may be included in neural field models 
such as the time scale of synaptic response or delays due to finite conduction speed of spikes along fibers. However, it was shown that neural field models may not be able to reflect accurately a behavior depending on complex local topology of neuron connections [11], but for relatively coarse behaviours, such as the regular bursting that occurs in PD in which neural masses behave as a unit, such models should be suitable.

Overall, neural field models deal with spatially extended networks, include various connectivity patterns, may include time delays, and allow analytical treatment. Thus, neural field models are appropriate to investigate new stimulation patterns especially designed to suppress brain rhythms associated with various symptoms. Neural field models may represent an innovative solution for new medical applications.

\subsection{Selective, adaptive, closed-loop stimulation waveform}

Closed-loop stimulation is not a new idea, since S. A. Chkhenkeli [9] conceived the first closedloop stimulation device designed to alleviate epilepsy in the 1970s. Closed-loop control of epilepsy is also the focus of modeling studies [18]. Until now, no such closed-loop system is available for the clinical treatment of PD. One important objective for the refinement of DBS in PD is to close the loop in order to optimize clinical outcome and save battery. This is the purpose of the following method which also has the advantage of being less invasive than implantation of electrodes in the deep brain. In PD, neural activity in the primary motor cortex (M1) is found to be particularly active around a frequency of $10 \mathrm{~Hz}$ [48]. This frequency is twice the frequency of tremor $(5 \mathrm{~Hz})$, and it has been hypothesized that agonist and antagonist muscles are sequentially activated by M1, giving rise to $5 \mathrm{~Hz}$ tremor ("flip-flop" hypothesis [48]). It was shown that the presence of neural activity in this frequency band was highly correlated with tremor [48, 49], and that STN DBS durably reduces M1 activity in this frequency band and results in a drastic reduction in rest tremor. Furthermore, a recent study combining tremor recording and neural signal recording using a DBS electrode suggested that tremor was driven by subcortical (e.g., BG) and cortical (e.g., M1) neural signals [4]. Consequently, it appears that appropriate electrical stimulation of M1 may be effective in decreasing tremor by attenuating cortical neural activity in a frequency band centered around 10 $\mathrm{Hz}$, that we term the "pathological band" in the following.

We propose the following constraints for a new electrical stimulation method of M1 able to alleviate tremor in patients with PD:

- The stimulation signal should attenuate neural activity only in the "pathological" frequency band, and minimize inteference with physiological brain rhythms. This imposes a criterion of frequency selectivity.

- The stimulation signal should optimally adapt to the spatial distribution of the pathological frequency band, i.e., the stimulation signal could be different at several points of space, which imposes a criterion of spatial selectivity.

- The stimulation signal should tend toward 0 when the activity in the pathological band vanishes, which means that the stimulation signal must be adaptive, and function in a closed 
loop manner in real time (sequentially recording the system state and then stimulating the system).

- Ideally, the stimulation signal should adapt to incoming activity from other structures projecting to the stimulation target, which imposes a criterion of robustness.

To achieve this, we propose the following. Let $\alpha(\mathbf{r}, t)$ be the closed-loop stimulation term applied at position $\mathbf{r}$ at time $t$. The objective is to find an analytical expression of $\alpha(\mathbf{r}, t)$ such that 1) the constraints mentioned above are taken into account, and 2) it effectively suppresses or attenuates predetermined frequency bands of neuronal activity associated with the presence of a symptom (i.e., the $10 \mathrm{~Hz}$ in M1 associated with rest tremor in PD). Let us assume that neural activity in the sub-part of M1 in which the pathological band is present may be described by the following neural field equation:

$$
\hat{L}[\psi(\mathbf{r}, t)]=\gamma \int_{\Omega} d \mathbf{r}^{\prime} W\left(\mathbf{r}-\mathbf{r}^{\prime}\right) S\left[\psi\left(\mathbf{r}^{\prime}, t-\frac{\left|\mathbf{r}-\mathbf{r}^{\prime}\right|}{c}\right)\right]+I(\mathbf{r}, t)
$$

where $I(\mathbf{r}, t)$ is the sum of external inputs (unknown a priori), which may include projections from the premotor cortex (PM) or the thalamus. Let us note that $\mathbf{r}$ represents a position in a $\mathrm{N}$ dimensional space, e.g. $N=2$. Then, we write $\psi(\mathbf{r}, t)$ in terms of a wave packet:

$$
\psi(\mathbf{r}, t)=\varphi(\mathbf{r}, t)+\frac{1}{\sqrt{2 \pi}} \int d \mathbf{k} \int_{\omega^{\prime}}^{\omega^{\prime \prime}} d \omega \tilde{\psi}(\mathbf{k}, \omega) e^{i(\omega t-\mathbf{k} . \mathbf{r})}
$$

where

$$
\varphi(\mathbf{r}, t)=\frac{1}{\sqrt{2 \pi}} \int d \mathbf{k}\left[\int_{-\infty}^{\omega^{\prime}} d \omega \tilde{\psi}(\mathbf{k}, \omega) e^{i(\omega t-\mathbf{k} . \mathbf{r})}+\int_{\omega^{\prime \prime}}^{+\infty} d \omega \tilde{\psi}(\mathbf{k}, \omega) e^{i(\omega t-\mathbf{k} . \mathbf{r})}\right]
$$

is the desired solution to the neural field equation, $\tilde{\psi}$ being the Fourier transform of $\psi$ (transformed in space and time). In other words, $\varphi(\mathbf{r}, t)$ represents a neural field in which the temporal modes such that $\omega \in\left[\omega^{\prime}, \omega^{\prime \prime}\right]$, where $\omega=2 \pi f$ (and $f$ is the frequency), are suppressed.

If we insert this into the neural field equation of $\psi(\mathbf{r}, t)$, and assume that we may linearize the sigmoid such as $S(x+y) \approx 0.5 S(2 x)+0.5 S(2 y)^{\|}$, then it gives:

$\hat{L}[\psi(\mathbf{r}, t)]=\frac{\gamma}{2} \int_{\Omega} d \mathbf{r}^{\prime} W\left(\mathbf{r}-\mathbf{r}^{\prime}\right) S\left[2 \varphi\left(\mathbf{r}^{\prime}, t-\frac{\left|\mathbf{r}-\mathbf{r}^{\prime}\right|}{c}\right)\right]+\frac{\gamma}{2} \int_{\Omega} d \mathbf{r}^{\prime} W\left(\mathbf{r}-\mathbf{r}^{\prime}\right) S\left[2 \zeta\left(\mathbf{r}^{\prime}, t-\frac{\left|\mathbf{r}-\mathbf{r}^{\prime}\right|}{c}\right)\right]+I(\mathbf{r}, t)$

with $\zeta(\mathbf{r}, t)=\frac{1}{\sqrt{2 \pi}} \int d \mathbf{k} \int_{\omega^{\prime}}^{\omega^{\prime \prime}} d \omega \tilde{\psi}(\mathbf{k}, \omega) e^{i(\omega t-\mathbf{k} . \mathbf{r})}$ is the pathological frequency band.

\footnotetext{
"This may be justified by the following: the sigmoid may be also expressed using the tanh function using $S(x)=$

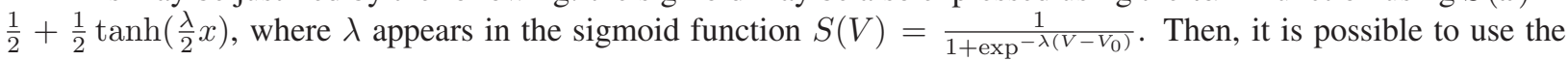
first-order approximation $\tanh (x) \approx x$, valid for moderate values of $x$ (but not for extreme values).
} 
Let us note that this function $\zeta(t)$ is not an abstract object: indeed, it can be recorded with implanted microelectrodes, and extracted from the recorded membrane potential by a signal processing chipset. However, undesirable frequencies may arise not only from intrinsic network properties such as connectivity (synaptic weights, spatial range of fibers, delays) but also from external factors, the most obvious being the input $I(\mathbf{r}, t)$. Thus, it is also useful to write the input as the sum of a "physiological" part $\kappa(\mathbf{r}, t)$ and of a "parkinsonian" part $\zeta_{I}(\mathbf{r}, t)$ :

$$
I(\mathbf{r}, t)=\kappa(\mathbf{r}, t)+\zeta_{I}(\mathbf{r}, t)
$$

where $\zeta_{I}(\mathbf{r}, t)=\frac{1}{\sqrt{2 \pi}} \int_{\omega^{\prime}}^{\omega^{\prime \prime}} d \omega \tilde{I}(\mathbf{r}, \omega) e^{i \omega t-\mathbf{k . r}}$ corresponds to the undesirable part of the input. Thus, we may write:

$$
\hat{L}[\psi(\mathbf{r}, t)]=\frac{\gamma}{2} \int_{\Omega} d \mathbf{r}^{\prime} W\left(\mathbf{r}-\mathbf{r}^{\prime}\right) S\left[2 \varphi\left(\mathbf{r}^{\prime}, t-\frac{\left|\mathbf{r}-\mathbf{r}^{\prime}\right|}{c}\right)\right]+\kappa(\mathbf{r}, t)+\eta(\mathbf{r}, t)
$$

where $\eta(\mathbf{r}, t)=\frac{\gamma}{2} \int_{\Omega} d \mathbf{r}^{\prime} W\left(\mathbf{r}-\mathbf{r}^{\prime}\right) S\left[2 \zeta\left(\mathbf{r}^{\prime}, t-\frac{\left|\mathbf{r}-\mathbf{r}^{\prime}\right|}{c}\right)\right]+\zeta_{I}(\mathbf{r}, t)$ is a spurious term due to the undesirable component of neuronal activity arising from intrinsic and extrinsic network properties.

Overall, the external and internal contributions potentially giving rise to the undesirable rhythm may be suppressed accordingly by adding an extra control term $\alpha(\mathbf{r}, t)$, such as

$$
\alpha(\mathbf{r}, t)=-\eta(\mathbf{r}, t)=-\frac{\gamma}{2} \int_{\Omega} d \mathbf{r}^{\prime} W\left(\mathbf{r}-\mathbf{r}^{\prime}\right) S\left[2 \zeta\left(\mathbf{r}^{\prime}, t-\frac{\left|\mathbf{r}-\mathbf{r}^{\prime}\right|}{c}\right)\right]-\zeta_{I}(\mathbf{r}, t)
$$

where $\alpha(\mathbf{r}, t)$ is the closed-loop stimulation term, that is a function of measured potential values throughout the neural field at the current time, but also in the past. The complete equation of the cortical target receiving the closed-loop stimulation is thus:

$$
\hat{L}[\psi(\mathbf{r}, t)]=\frac{\gamma}{2} \int_{\Omega} d \mathbf{r}^{\prime} W\left(\mathbf{r}-\mathbf{r}^{\prime}\right) S\left[2 \varphi\left(\mathbf{r}^{\prime}, t-\frac{\left|\mathbf{r}-\mathbf{r}^{\prime}\right|}{c}\right)\right]+\kappa(\mathbf{r}, t)
$$

In this case, the neural field equation describes the evolution of the neural field $\psi(\mathbf{r}, t)$ in which the pathological band is present, towards the neural field $\varphi(\mathbf{r}, t)$ where this pathological band is absent. This evolution rate is $\frac{1}{\beta}$ in the case of a first-order temporal operator. Thus, undesirable frequency components are progressively damped out until their disappearance. It should be noted that this control term is general in that it does not depend of the temporal operator $\hat{L}$ (that may be of second order).

One should note that, with such a stimulation signal, only the undesired brain rhythm will be affected by the stimulation signal (frequency selectivity). Then, the stimulation signal is different at each spatial position, which satisfies the constraint of spatial selectivity. Furthermore, if the undesirable rhythm or input vanishes, i.e. $\zeta(\mathbf{r}, t) \rightarrow 0$ or $\zeta_{I}(\mathbf{r}, t) \rightarrow 0$, then the control term $\alpha(\mathbf{r}, t)$ tends towards 0 , which means that, as required, the stimulation signal is adaptive. Finally, this term adapts to fluctuations in the input by constantly filtering it, which fulfills the robustness criterion. Overall, the closed-loop stimulation method stimulates only where and when needed, taking into account the connectivity within the network, past activity of the network and finite conduction delays of spikes within the network. 


\section{Numerical results for a spiking neuron network model}

\subsection{Adapting the control term for a spiking neuron network model}

The closed-loop stimulation signal designed to specifically suppress neural activity in predetermined frequency bands has been derived using a neural field model, in which the basic unit is the neural mass. In the following, we explore the response of a single neural mass, under the form of a network of several hundreds of excitatory and inhibitory cortical neurons, to our closed-loop stimulation method. However, in order to evaluate the effect of this closed-loop stimulation method on a network of spiking neurons, several adjustments have to be made. Let us consider the control term proposed above using our neural field model:

$$
\alpha(\mathbf{r}, t)=-\eta(\mathbf{r}, t)=-\frac{\gamma}{2} \int_{\Omega} d \mathbf{r}^{\prime} W\left(\mathbf{r}-\mathbf{r}^{\prime}\right) S\left[2 \zeta\left(\mathbf{r}^{\prime}, t-\frac{\left|\mathbf{r}-\mathbf{r}^{\prime}\right|}{c}\right)\right]-\zeta_{I}(\mathbf{r}, t)
$$

First, we assume that one single electrode stimulates the network of $N$ neurons, and that every neuron will receive the same input current. Second, the mean membrane potential $V(t)=$ $\frac{1}{N} \sum_{i=1}^{N} v_{i}(t)$ of the network is monitored to detect the presence of pathological rhythms, implying that only the temporal modes $\omega$ may be detected (and not the spatial modes k). Third, let $\tilde{\zeta}_{I}(t)$ be the undesirable component of the total input to the network, i.e., synaptic inputs within the network and external inputs from other neural networks, which is equivalent to $-\gamma \int_{\Omega} d \mathbf{r}^{\prime} W(\mathbf{r}-$ $\left.\mathbf{r}^{\prime}\right) S\left[\zeta\left(\mathbf{r}^{\prime}, t-\frac{\left|\mathbf{r}-\mathbf{r}^{\prime}\right|}{c}\right)\right]-\zeta_{I}(\mathbf{r}, t)$ in the neural field model. The simplified control signal $C(t)$ derived from the neuronal field model is:

$$
C(t)=-\tilde{\zeta}_{I}(t)
$$

which has to be applied identically to each neuron of the spiking network. The physical meaning behind this control term is that the total input to the network (external and internal synaptic contributions) is "filtered" by an appropriate recording and stimulated accordingly to suppress undesirable frequencies.

\subsection{Simulation of a spiking neuron network model with closed-loop stimu- lation}

We designed a model at a microscopic scale, where units are neurons. These neurons are randomly connected in a two-dimensional network. For each excitatory neuron's connection, an axonal conduction delay $\Delta$ is implemented (Eq. 3.4). This delay between the presynaptic neuron's spike and the receiving of the signal by the postsynaptic neuron depends on the distance (Eq. 3.3) between these two connected neurons.

$$
\begin{aligned}
d_{i j} & =\sqrt{\left(x_{i}-x_{j}\right)^{2}+\left(y_{i}-y_{j}\right)^{2}} \\
\Delta_{i j} & =\frac{d_{i j}}{c}
\end{aligned}
$$


In Eq. 3.4, $c$ is the speed of axonal conduction chosen as a function of the maximum delay and the maximum distance between neurons in the network (3.5).

$$
\begin{aligned}
c & =\frac{d_{\max }}{\Delta_{\max }} \\
d_{\max } & =\sqrt{\left(1-X_{\max }\right)^{2}+\left(1-Y_{\max }\right)^{2}}
\end{aligned}
$$

This combination of realistic cellular patterns and axonal conduction delay allows emergence of biologically observed activity patterns, such as polychronization [25] or oscillations at specific bandwidths. At each time step, the membrane potential of neurons is calculated according to the differential equations (Eq. 3.7, 3.8) described in the Izhikevich model [24]:

$$
\begin{aligned}
& v^{\prime}=0.04 v^{2}+5 v+140-u+I \\
& u^{\prime}=a(b v-u)
\end{aligned}
$$

with an after-spike resetting :

$$
\text { if } v \geq 30 \mathrm{mV} \text {, then }\left\{\begin{array}{l}
v \leftarrow c \\
u \leftarrow u+d
\end{array}\right.
$$

where $v$ describes the membrane potential, $u$ describes the membrane recovery variable, $a$ determines the speed of membrane recovery (higher values of $a$ result in a faster recovery), $b$ determines the sensitivity of $u$ to the subthreshold oscillations of $v, c$ is the after-spike reset value of $v, d$ determines the after-spike reset value of $u$.

This model of spiking neurons allows fast computation and is able to reproduce the activity of different types of cortical neurons (among others), such as fast spiking neurons or intrinsically bursting neurons [24].

To observe the pathological activity in the network, we simulate input from the premotor cortex by introducing a $10 \mathrm{~Hz}$ bursting input to the network. These inputs are sent to a different random selection of neurons at each time step. In the spiking neuron network model, the time step used was $1 \mathrm{~ms}$, giving rise to a maximum possible sampling frequency of $1000 \mathrm{~Hz}$. At each time step, a segment of the last $100 \mathrm{~ms}$ (i.e., the last 10 voltage values computed, last $100 \mathrm{~ms}$, since the chosen sampling frequency was $F_{s}=100 \mathrm{~Hz}$ ) of the mean membrane potential is extracted and filtered using a low-pass filter. In this simulation, the cut-off frequency for this low-pass filter is fixed at $11 \mathrm{~Hz}$. Only the last point of the resulted segment, corresponding to the current state of the mean membrane potential, is recorded. Indeed, the filtered segments overlap (since a segment is extracted every time step and not every $100 \mathrm{~ms}$ ), consequently implying that the first points of a segment would be redundant with the previous computed segment.

In order to minimize the artificial noise introduced by this method, we calculate the mean between the recorded point and the 10 last points of the filtered estimated input. This results in a smoothed signal in which only the $10 \mathrm{~Hz}$ frequency component is predominant, representing the abnormal contribution of the network membrane potential. Assuming that we measure the mean 
potential $\bar{v}$ of the network, we then use the following equation for the $v$ variable [24] to compute the total input to the network (synaptic and eternal inputs):

$$
\bar{v}(t)=0.04 \bar{v}^{2}(t)+5 \bar{v}(t)+140-\bar{u}(t)+I(t)
$$

where the mean network variables are $(\bar{v}, \bar{u})$. This gives immediately $I(t)=\bar{v}(t)-0.04 \bar{v}^{2}(t)-$ $5 \bar{v}(t)-140+\bar{u}(t)$. One issue is that the variable $u$ has no physical meaning and is not measurable. Thus, we considered that $\bar{u}(t) \approx \bar{u}_{0}$ along the simulation, i.e., the mean value of $u$ for the network does not vary much during the simulation. We checked after simulation that this approximation was verified, with values of $\bar{u}$ lying between -10.5 and $-13.5 \mathrm{mV}$. Finally, this filtered signal of the total input to the network is inserted at the end of the filtered estimated input segment and the stimulation signal is computed as the negative of this segment. In other terms, the stimulation signal is directly computed from the filtered estimated input to the network.

The simulation is on 10 seconds and the network is parameterized in order to produce a 10 $\mathrm{Hz}$ pathological signal. This $10 \mathrm{~Hz}$ signal is detected by our filtering analysis and the stimulation computed accordingly to Eq. 3.2 is sent to the network during two periods (between $t=1-5$ and $t=6-10 \mathrm{~s}$ ). The mean potential of the network (simply computed as the average on all membrane potential values) is shown in Fig. 4.

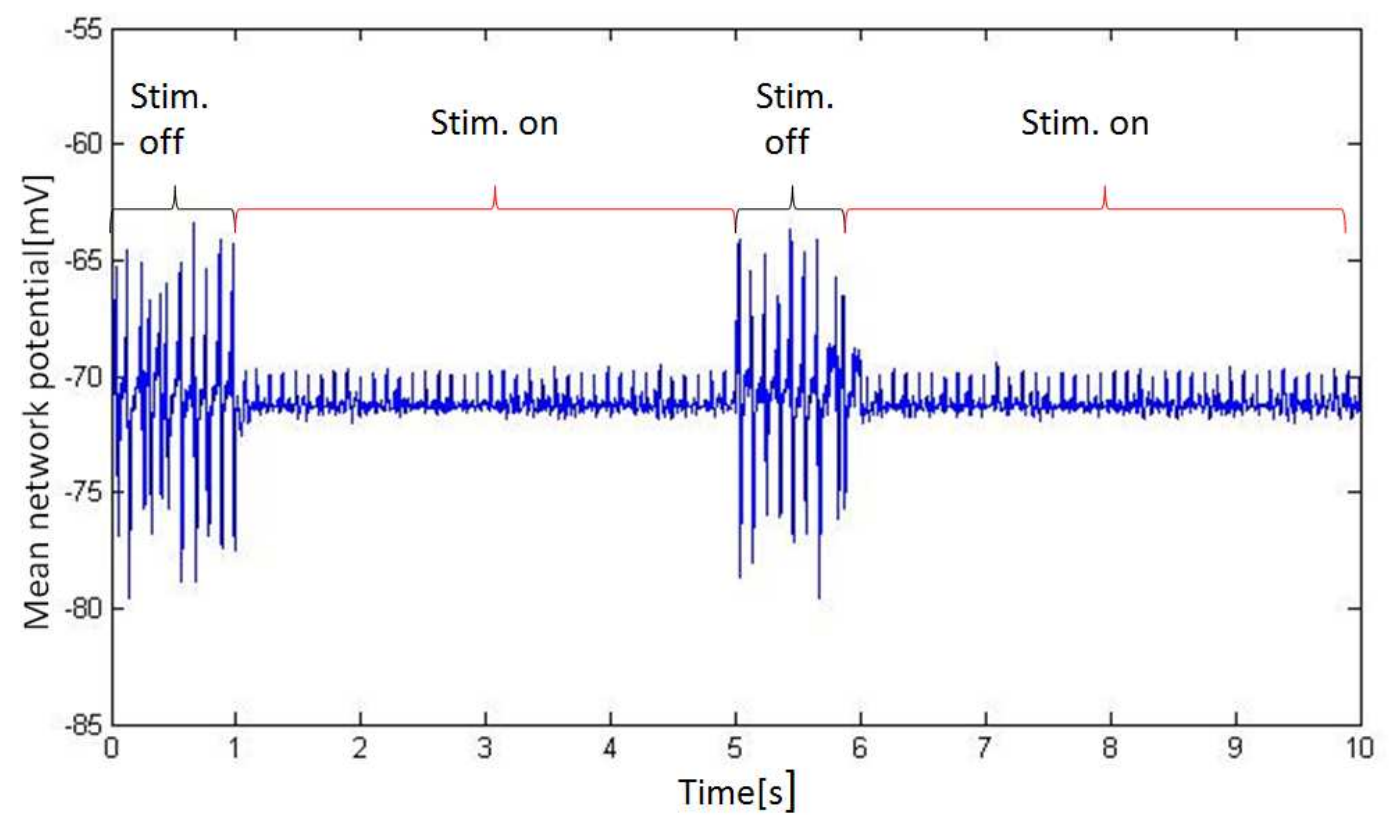

Figure 4: Network activity without $(0-1$ and $5-6 \mathrm{~s})$ and during $(1-5$ and $6-10 \mathrm{~s})$ closed-loop stimulation.

The strong oscillations in the mean potential correspond to the $10 \mathrm{~Hz}$ frequency caused by the incoming $10 \mathrm{~Hz}$ bursts simulating input from the premotor cortex. When the stimulation signal is present $(1-5$ and $6-10 \mathrm{~s})$, we observe that these strong $10 \mathrm{~Hz}$ oscillations rapidly disappear. We observe that, when the stimulation is turned "off" at time $t=5 \mathrm{~s}$, the $10 \mathrm{~Hz}$ activity re-appears 
rapidly due to incoming bursting activity. When stimulation is turned "on" again, this undesirable rhythm is also effectively attenuated. From these simulation results, we conclude that these oscillations, correlated with the presence of tremor in human patients, are durably attenuated throughout the simulation, confirming that our closed-loop stimulation method is effective in detecting the undesirable rhythm and appropriately stimulating the network. Possible improvements are discussed in the following section.

\section{Discussion and concluding remarks}

Recent interpenetration between neuroscience, mathematics and physics combined with technological progress has profoundly influenced our understanding of physiological mechanisms underlying brain neuromodulation. Here we review deep brain stimulation, a standard therapeutic strategy used to alleviate symptoms in Parkinson's disease developed more than 20 years after the crucial observations made by Jasper in Montreal. We show that, over the last decade, a variety of computational models were proposed, focusing at different spatial scales (cellular - spiking neuron network models, network - phase oscillator models).

Importantly, theoretical predictions must be validated by experimental and clinical studies. One example is the idea of resonance in the subthalamo-cortical circuits in PD first hypothesized by Modolo et al. [34] and recently confirmed by Eusebio and co-workers [13]. Future modeling studies will probably be based on such mesoscopic approaches. From this perspective, we propose a neural field model which provides a quantitative description of continuous cortical activity in space and time. One of the assumptions made in this theoretical approach is that brain activity can be adequately described by neural field equations. Interestingly, neural field models have not been used in brain neuromodulation applications yet, and we propose to use them to explore the attenuation or suppression of undesirable rhythms present in the cortex and associated with the presence of PD symptoms. We show that neural field models may be helpful in exploring new selective, adaptive, closed-loop stimulation waveforms and anticipate that these theoretical developements will lead to new stimulation protocols and limit the need for animal testing ("Reduce, Refine, Replace" -3R's- [40]). From the practical point of view, cortical stimulators of the type we propose here would offer advantages over DBS as currently implemented, such as a limited invasiveness.

However, the hypotheses we put forward to derive our control term for a neural field model present some limitations. Strictly speaking, the voltage signal should be stationary to allow Fourier decomposition and filtering at each time, which is not true for real brain signals. However, it is possible to consider the Fourier series on a short time interval. Another possibility would be to write the solution under the form of a wavelet transform, which would free us from the stationarity condition. There is also room for improvement regarding the linear approximation of the sigmoid function, and perturbation theory may be used to improve the approximation of the integral term. Finally, we assume that the injected current is identical to those received by neural masses within the network, which is likely to be different in a biological situation, because of inhomogeneous resistivity in brain tissue, orientation of fibers, or stimulated elements (cellular body or axon). Thus, further work is needed to establish a reliable "transfer function" allowing us to know the 
optimal current to apply to obtain the desired current received at the neuronal level at the cellular level computed by our method, which may be implemented by using approaches similar to that of McIntyre and coworkers [30].

The results of this study call for several improvements. First, the addition of an external bursting input at a frequency of $5 \mathrm{~Hz}$ in our spiking neuron network, simulating activity received by M1 from the thalamus in PD, may be an opportunity to evaluate the robustness of the proposed stimulation method. Second, the control term could be tested numerically in a two-dimensional neural field model with first- and second-order temporal operators in order to verifiy if undesirable frequency components are suppressed not only in time, but also in space. Indeed, our current spiking neuron network does include space, but the numerical stimulation we used is the same for all neurons in the network. Third, one possible candidate to detect frequency fluctuations is the use of a "Phase-Locked Loop" circuit (PLL), commonly used in electronics or control theory in order to monitor frequency fluctuations between a reference signal and a signal in which the frequency may be changed. In our case, the reference signal may be the measured neural signal, in order to adapt the frequency of the stimulation signal. Fourth, it might be useful to investigate if adding in the control term a function of $\hat{L}[\zeta(\mathbf{r}, t)]$ accelerates the convergence speed between the measured field $\psi(\mathbf{r}, t)$ and the desired field $\varphi(\mathbf{r}, t)$. Indeed, it may help to control intrinsic oscillations of neural masses. These possibilites are currently explored and will be the object of forthcoming studies.

Results presented in this paper illustrate how theoreticians and neuroscientists can work "hand in hand", back and forth between disciplines, to build the foundations of new mechanism-based brain stimulation methods. One may ask whether the ultimate convergence point between neuroscience and mathematics for brain stimulation might be. Our stimulation method is based on a closed-loop feedback involving several spatial scales, controlling accordingly a cortical target activity with a matrix of micro-electrodes to provide stimulation. This matrix of micro-electrodes may be used to record as well as stimulate the cortical target, and this matrix may obey also a neural field equation. Since micro-electrodes interact with cortical tissue at the same spatial scale (sub-mm, mesoscopic), it would have the effect to add a supplementary network of cortical neurons from the "cortical point of view". Thus, the matrix of micro-electrodes may be seen as a "virtual cortical module" playing the role of a replacement part for the cortex when necessary. By doing so, both the cortex and the stimulator would speak the same language.

\section{Acknowledgements}

This work was supported by the European Network of Excellence BioSim (contract No. LSHBCT-2004-005137) to Anne Beuter, by a Post-Doctoral Research Fellowship from the Government of Canada (International Bureau for Canadian Education) to Julien Modolo, and by the Marie Curie "Research Training Network" Neurovers-IT to Basabdatta Bhattacharya. 


\section{References}

[1] C. Ajmone Marsan. Focal electrical stimulation. In: Experimental Models of Epilepsy: A manual for the laboratory worker. Eds D. P. Purpura, J. K. Penry, D. Tower, D. M. Woodbury and R. Walter, Raven Press, New York, 1972.

[2] S. Amari. Dynamics of pattern formation in lateral-inhibition type neural fields. Biol. Cybern., 27 (1977), No. 2, 77-87.

[3] F. Atay, A. Hutt. Stability and bifurcations in neural fields with finite propagation speed and general connectivity. SIAM J. Appl. Math., 65 (2005), No. 2, 644-666.

[4] U. B. Barnikol, O. V. Popovych, C. Hauptmann, V. Sturm, H. J. Freund, P. A. Tass. Tremor entrainment by patterned low-frequency stimulation. Philos. Transact. A Math. Phys. Eng. Sci., 366 (2008), No. 1880, 3543-3573.

[5] R. Bartolow. Experimental investigations into the functions of the human brain. AM. J. Med. Sci., 1874, 305-313.

[6] N. P. Bechtereva, A. N. Bondarchuk, V. M. Smirnov. Therapeutic electrostimulations of deep brain structures. Vopr Neirokhir, 1 (1972), 115-120.

[7] A. L. Benabid, P. Pollak, A. Louveau, S. Henry, J. de Rougemont. Combined (thalamotomy and stimulation) stereotactic surgery of the Vim thalamic nucleus for bilateral Parkinson disease. Appl. Neurophysiol., 50 (1987), No. 1-6, 344-346.

[8] A. L. Benabid, W. Bradley, J. Mitrofanis, C. Xia, B. Piallat, V. Fraix, A. Batir, P. Krack, P. Pollak, F. Berger. Therapeutic electrical stimulation of the central nervous system. C. R. Biologies, 328 (2005), 177-186.

[9] S. A. Chkhenkeli. Direct deep brain stimulation: first steps toward the feedback control of seizures. In: Epilepsy as a dynamical disease, p.249-262. Eds J. Milton and P. Jung, SpringerVerlag, New York, 2003.

[10] J. Echauz, H. Firpi, G. Georgoulas. Intelligent control strategies for neurostimulation. In: Applications of intelligent control of engineering systems. Ed P. K. Valavanis, Springer, 2009.

[11] R. Edwards. Approximation of neural network dynamics by reaction-diffusion equations. Math. Meth. App. Sci., 19 (1996), 651-677.

[12] G. B. Ermentrout, J. D. Cowan. A mathematical theory of visual hallucination patterns. Biol. Cybern., 34 (1979), No. 3, 137-150.

[13] A. Eusebio, A. Pogosyan, S. Wang, B. Averbeck, L. D. Gaynor, S. Cantiniaux, T. Witjas, P. Limousin, J. P. Azulay, P. Brown. Resonance in subthalamo-cortical circuits in Parkinson's disease. Brain, 132 (2009), No. 8, 2139-2150. 
[14] W. Gerstner, R. Kempter, J. L. van Hemmen, H. Wagner. A neuronal learning rule for submillisecond temporal coding. Nature, 383 (1996), 76-81.

[15] F. A. Gibbs, E. L. Gibbs, W. G. Lennox. The likeness of the cortical dysrhythmias of schizophrenia and psychomotor epilepsy. Am. J. Psychiatry, 95 (1938), 255-269.

[16] P. L. Gildenberg. History of electrical neuromodulation for chronic pain. Pain Medicine, 7 (2006), S7-S13.

[17] B. J. Gluckman, E. J. Neel, T. I. Neto, W. L. Ditto, M. L. Spano, S. J. Schiff. Electric field suppression of epileptiform activity in hippocampal slices. J. Neurophysiol., 6 (1996), 42024205.

[18] B. J. Gluckman, H. Nguyen, S. L. Weinstein, S. J. Schiff. Adaptive electric field control of epileptic seizures. J. Neurosci., 21 (2001), No. 2, 290-600.

[19] S. Grillner, A. Kozlov, J. H. Kotaleski. Integrative neuroscience: linking levels of analyses. Curr. Opin. Neurobiol., 15 (2005), No. 5, 614-621.

[20] R. Hassler, F. Mundiger, T. Riechert. Correlations between clinical and autoptic findings in stereotaxic operations in parkinsonism. Confin. Neurol., 26 (1965), 282-290.

[21] A. L. Hodgkin, A. F. Huxley. A quantitative description of membrane current and its application to conduction and excitation in nerve. J. Physiol., 117 (1952), No. 4, 500-544.

[22] J. C. Horton, D. L. Adams. The cortical column: a structure without a function. Phil. Trans. of the Royal Soc. B, 360 (2005), No. 1456, 837-862.

[23] X. Huang, W. C. Troy, Q. Yang, H. Ma, C. R. Laing, S. J. Schiff, J. Y. Wu. Spiral waves in disinhibited mammalian neocortex. J. Neurosci., 24 (2004), 9897-9902.

[24] E. M. Izhikevich. Simple model of spiking neurons. Transactions on Neural Networks, 14 (2003), 1569-1572.

[25] E. M. Izhikevich. Polychronization: computation with spikes. Neural Computation, 18 (2006), 245-282.

[26] H. H. Jasper. Recording from microelectrodes in stereotactic surgery for Parkinson's disease. J. Neurosurg., 24 (1966), 219-221.

[27] E. I. Kandel. Functional and stereotactic neurosurgery. Plenum Medical Book Co, New York, 1966.

[28] R. R. Llinas, U. Ribary, D. Jeanmonod, E. Kronberg, P. P. Mitra. Thalamocortical dysrhythmia: a neurological and neuropsychiatric syndrome characterized by magnetoencephalography. Proc. Natl. Acad. Sci. USA, 96 (1999), No 26, 15222-15227. 
[29] H. O. Lüders. Deep brain stimulation and epilepsy. Martin Dunitz, New York, 2004.

[30] C. C. McIntyre, S. Mori, D. L. Sherman, N. V. Thakor, J. L. Vitek. Electric field and stimulating influence generated by deep brain stimulation of the subthalamic nucleus. Clin. Neurophysiol., 115 (2004), No. 3, 589-595.

[31] W. Meissner, A. Leblois, D. Hansel, B. Bioulac, C. E. Gross, A. Benazzouz, T. Boraud. Subthalamic high frequency stimulation resets subthalamic firing and reduces abnormal oscillations. Brain, 128 (2005), No. 10, 2372-2382.

[32] J Milton, P. Jung. Epilepsy as a dynamical disease. Springer-Verlag, New York, 2003.

[33] J. Modolo, J. Henry, A. Beuter. Dynamics of the subthalamo-pallidal complex in Parkinson's disease during deep brain stimulation. J. Biol. Phys., 34 (2008), No. 3-4, 351-366.

[34] J. Modolo, A. Beuter. Contribution of cortical inputs to subthalamic activity during deep brain stimulation. Proceedings of the Neurocomp 2008 conference, Marseille, France (2008).

[35] J Modolo, A. Beuter. Linking brain dynamics, neural mechanisms and deep brain stimulation in Parkinson's disease: an integrated perspective. Med. Eng. Phys., 31 (2009), 615-623.

[36] D. Q. Nykamp, D. Tranchina. A population density approach that facilitates largescale modeling of neural networks : analysis and an application to orientation tuning. J. Comput. Neurosci., 8 (2000), No. 1, 19-50.

[37] J. Olszewski. The thalamus of the Macaca Mulatta. An atlas for use with the stereotactic instrument. Basel Karger, 1952.

[38] A. Omurtag, B. W. Knight, L. Sirovich. On the simulation of large populations of neurons. J. Comput. Neurosci., 8 (2000), No. 5, 51-63.

[39] A. Pascual, J. Modolo, A. Beuter. Is a computational model useful to understand the effect of deep brain stimulation in Parkinson's disease? J. Integr. Neurosci., 5 (2006), No. 4, 541-559.

[40] J. Richmond. The 3Rs-Past, present and future. Scand. J. Lab. Anim. Sci., 27 (2000), 84-92.

[41] J. E. Rubin, D. Terman. High frequency stimulation of the subthalamic nucleus eliminates pathological thalamic rhythmicity in a computational model. J. Comput. Neurosci., 16 (2004), No. 3, 211-235.

[42] D. Rubino, K. A. Robbins, N. G. Hatsopoulos. Propagating waves mediate information transfer in the motor cortex. Nature Neurosci., 9 (2006), No. 12, 1549-1557.

[43] J. D. Speelman, D. A. Bosch. Resurgence of functional neurosurgery for Parkinson's disease: a historical perspective. Mov. Disord., 13 (1998), No. 3, 582-588.

[44] E. A. Spiegel, H. T. Wycis, M. Marks, A. S. Lee. Stereotaxic apparatus for operations on the human brain. Science, 106 (1947), 349-350. 
[45] A. A. Spiegel, H. T. Wycis. Stereoencephalotomy (thalamic related procedures) part 1: Methods and atlas for the human brain. Grune and Stratton, New York, 1952.

[46] P. A. Tass. Phase Resetting in Medicine and Biology. Stochastic Modelling and Data Analysis. Series: Springer Series in Synergetics, 1999.

[47] D. Terman, J. E. Rubin, A. C. Yew, C. J. Wilson. Activity patterns in a model for the subthalamopallidal network of the basal ganglia. J. Neurosci., 22 (2002), No. 7, 2963-2976.

[48] L. Timmermann, J. Gross, M. Dirks, J. Volkmann, H. J. Freund, A. Schnitzler. The cerebral oscillatory network of parkinsonian resting tremor. Brain, 126 (2003), No. 1, 199-212.

[49] L. Timmermann, E. Florin, C. Reck. Pathological cerebral oscillatory activity in Parkinson's disease: a critical review on methods, data and hypotheses. Expert Rev. Med. Dev., 4 (2007), No 5, 651-61.

[50] M. S. Titcombe, L. Glass, D. Guehl, A. Beuter. Dynamics of Parkinsonian tremor during deep brain stimulation. Chaos, 11 (2001), No. 4, 766-773.

[51] J. L. P. Velazquez. Brain, behaviour and mathematics: Are we using the right approaches? Physica D, 212 (2005), 161-182.

[52] J. A. Vilensky, S. Gilman. Horsley was the first to use electrical stimulation of the human cerebral cortex intraoperatively. Surg. Neurol., 58 (2002), 425-426.

[53] H. R. Wilson, J. D. Cowan. A mathematical theory of the functional dynamics of cortical and thalamic nervous tissue. Kybernetik, 13 (1973), No. 2, 55-80.

[54] T. Wichmann, M. R. Delong. Deep brain stimulation for neurologic and neuropsychiatric disorders. Neuron, 52 (2006), No. 1, 197-204.

[55] A. Winfree. Are cardiac waves relevant to epileptic waves propagation? In: Epilepsy as a dynamical disease, p. 165-188. Eds J. Milton and P. Jung, Springer-Verlag, New York, 2003.

[56] J. S. Yeomans. Principles of Brain Stimulation. Oxford University Press, New York, 1990. 